\title{
CORRELATED HOPPING MODEL FOR SINGLET EXCITON TRANSPORT IN LIGHTLY DOPED NAPHTHALENE CRYSTALS
}

\author{
Panos ARGYRAKIS and Raoul KOPELMAN \\ Department of Chemistry, The University of Michigan, Ann Arbor, Michigan 48109, USA
}

Received 28 January 1980; in final form 15 May 1980

\begin{abstract}
A formalism of correlated lattice random walk is applied to the singlet exciton transport in naphthalene. Fluorescence experiments on excitation migration in naphthalene crystals $\left(\mathrm{C}_{10} \mathrm{H}_{8}\right.$ and $\mathrm{C}_{10} \mathrm{D}_{8}$ ), lightly dopcd chemically (anthracene) or isotopically $\left(\mathrm{C}_{10} \mathrm{DH}_{7}\right)$, over a wide temperature range $(2-300 \mathrm{~K})$ are interpreted in terms of a correlated hopping model. The hopping time is found to be of the order of 1 ps. The increase in the overall quenching (supertrapping) efficiency with reduced temperature is interpreted in terms of an increase by one or more orders of magnitude in the parameter of coherency (hopping correlation). The role of the trapping cross section is discussed.
\end{abstract}

\section{Introduction}

Crystals doped with impurities, lightly or heavily, have lately been used to a large extent in electronic energy transport studies [1-4]. Heavily doped sys. tems used by us $[5,6]$ in connection with "exciton percolation" and "exciton coherence" exhibit critical concentration regions and associated critical phenomena. Lightly doped crystals, on the other hand, are simpler. The guest concentration here is at least five orders of magnitude lower than that of the main species, the host. Since the guest sites act as trapping sites they compete with the host for excitonic energy. The extent of energy transport, given in steady-state experiments via the guest/host fluorescence ratio, depends upon excitation exchange interactions, the lifetime, the host-guest energy difference, the temperature, other system impurities, etc. We focus on naphthalene crystals that were lightly doped with one of several different guests. We use the hopping model for the site-to-site transfer because we already have extensive information available [6-9] for hoppinglike random walks on lattices. It should be noted here that it is not necessary to describe the transfer phenomenon as a series of exactly defined hops per se. It is enough to assume that there are random

\footnotetext{
$\stackrel{\div}{\div}$ Supported by NIH Grant 2R01 NS08116-10A1.
}

scattering events that control the instantaneous position and direction of the energy wave packet and its mean frce path. Similarly, it is not clear (but it is rather unimportant regarding our results) what is defined as hopping time: the time of the actual hop (as a classical mechanics picture), or the time the excitation is localized on a particular site right before or after a hop (a quantum mechanical picture), or perhaps a combination of both?

We find that for the lowest singlet excitation of the pure naphthalene crystal a minimum of $10^{4}$ distinct molecular sites are visited (on the average) during the lifetime of a singlet excitation, while a typical value is rather 3 or 4 times this minimum. Recently several approaches have been used that are akin to correlated random walks. Kenkre and Knox $[10]$ made use of a generalized master equation to derive a memory function from experimental fluorescence and absorption lineshapes, providing short time information for different temperatures. Similar theoretical approaches have also been used by Silbey [3, 11] and by Haken et al. [12,13]. Several studies in connection with experiments $[14,15]$ were also reported. A new model was recently proposed by us [9] that extends calculations to multicomponent systems. We use a correlated random walk approach that relates the number of hops to the distinct number of sites visited and includes the retention of directional 
memory ("coherency") as a parameter. We show how one can use this approach to estimate the average number of "hops" that are performed by a generated excited state until its decay. We also show that the enhancement of energy transfer with decreasing temperature can be interpreted in terms of increased coherency.

\section{Experimental information}

The spectra of the following systens have been reported elsewhere: (a) anthracene in naphthalene, (b) partially deuterated naphthalenes in naphthalene $\mathrm{C}_{10} \mathrm{H}_{8}$. The relative fluorescence intensities are given in tables 1 and 2 , where $C_{\mathrm{g}}$ is the concentration of the guest, $Q_{\mathrm{g}} / Q_{\mathrm{h}}$ is the observed emission quantum ratio, and $P$ is defined as $q_{\mathrm{g}} /\left(q_{\mathrm{g}} \div q_{\mathrm{h}}\right)$, i.e. it is the fraction of excitation carried by the guest species (see appendix). The quantities $q$ are the quantum ratio $Q$ corrected by the quantum efficiency factors [16-19] $\phi($ i.e. $q=Q / \phi)$, and $\phi_{\mathrm{A}}=0.96$ and $\phi_{\mathrm{N}}=0.37$. Table I is based on data summarized by Wolf and Port [2] for anthracene in naphthalene, while table 2 refers to the deuterated naphthalene systems investigated in this laboratory by F.W. Ochs.

Figs. 1 and 2 show some of these spectra for illustration purposes. In fig. 1 the fluorescence spectrum of the $9.2 \times 10^{-5} 2-D_{1} / D_{3}$ system is shown for the "0-0" emission. Each feature is identified and all emission contributions are taken into account. In fig. 2 the $8.8 \times 10^{-4} 1-\mathrm{D}_{1} / \mathrm{D}_{8}$ system fluorescence

Table 1

Fluorescence quantum yields for the anthracene in naphthalene system - $300 \mathrm{~K}$

\begin{tabular}{lll}
\hline$C_{\mathrm{g}}$ & $Q_{\mathrm{g}} / Q_{\mathrm{h}}$ & $P$ \\
\hline $1 \times 10^{-5}$ & 0.80 & 0.24 \\
$7 \times 10^{-6}$ & 0.65 & 0.20 \\
$4 \times 10^{-6}$ & 0.40 & 0.13 \\
$2 \times 10^{-6}$ & 0.26 & 0.09 \\
$1 \times 10^{-6}$ & 0.17 & 0.06 \\
$7 \times 10^{-7}$ & 0.12 & 0.04 \\
$4 \times 10^{-7}$ & 0.08 & 0.03 \\
$1 \times 10^{-7}$ & 0.03 & 0.01 \\
$5 \times 10^{-8}$ & 0.02 & 0.007 \\
$3 \times 10^{-8}$ & 0.01 & 0.004
\end{tabular}

Table 2

Fluorescence quantum yields for the isotopic mixed naphthalene systems $-1.8 \mathrm{~K}$

\begin{tabular}{llll}
\hline Species a) & $\begin{array}{l}C_{\mathrm{g}} \\
\text { (mole fraction) }\end{array}$ & $Q_{\mathrm{g}} / Q_{\mathrm{h}}$ & $p^{\mathrm{b})}$ \\
\hline 1 & $8.8 \times 10^{-4}$ & 180 & 0.99 \\
2 & $8.8 \times 10^{-4}$ & 157 & 0.99 \\
3 & $9.2 \times 10^{-5}$ & 31 & 0.97 \\
\hline
\end{tabular}

a) Species 1: 1- $\mathrm{D}_{1} \mathrm{C}_{10} \mathrm{H}_{7}$ in $\mathrm{D}_{8}$, "0-512" band; species 2 : $1-\mathrm{D}_{1} \mathrm{C}_{10} \mathrm{H}_{7}$ in $\mathrm{D}_{8}$, " $0-0$ " band; species $3: 2-\mathrm{D}_{1} \mathrm{C}_{10} \mathrm{H}_{7}$ in $\mathrm{D}_{8}$, " $0-512$ " band.

b) We have assumed here equal quantum efficiencies and Franck-Condon factors.

Table 3

Temperature dependence of emission, anthracene in naphthalene $\left(C_{\mathrm{g}}=3.5 \times 10^{-5}\right)$

\begin{tabular}{|c|c|c|c|c|}
\hline \multirow[t]{2}{*}{$T(\mathrm{~K})$} & \multicolumn{2}{|c|}{ Hammer [17] } & \multicolumn{2}{|c|}{ Auweter [19] } \\
\hline & $Q_{\mathrm{g}} / Q_{\mathrm{h}}$ & $P$ & $k_{W G}$ & $P$ \\
\hline 2 & 9.83 & 0.79 & $1.2 \times 10^{8}$ & 0.86 \\
\hline 25 & 6.81 & 0.72 & $8.1 \times 10^{7}$ & 0.80 \\
\hline 50 & 4.33 & 0.63 & $4.3 \times 10^{7}$ & 0.68 \\
\hline 100 & 2.96 & 0.53 & $2.4 \times 10^{7}$ & 0.54 \\
\hline 150 & 2.54 & 0.49 & $1.8 \times 10^{7}$ & 0.47 \\
\hline 200 & 2.32 & 0.48 & $1.3 \times 10^{7}$ & 0.39 \\
\hline 300 & 2.01 & 0.44 & $9.2 \times 10^{6}$ & 0.31 \\
\hline
\end{tabular}

spectrum is shown for the " $0-512$ " $\mathrm{cm}^{-1}$ vibronic emission.

In table 3 we report the results given earlier by Hammer and Wolf $[17,18]$ and by Auweter [19], which show the quantum ratio of fluorescence as a function of temperature for anthracene in naphthalene. The transformation of Auweter's "rate constant" $k_{\text {WG }}$ into $P$ is described in the appendix. Note that we do not require $k_{W G}$ to be a real rate constant (time independent).

\section{Model description and calculations}

As mentioned earlier we assume here a generalized hopping model. Usually the concentration of the guest sites is much smaller compared to the host sites:

$C_{\mathrm{h}} \gg C_{\mathrm{g}}$ 


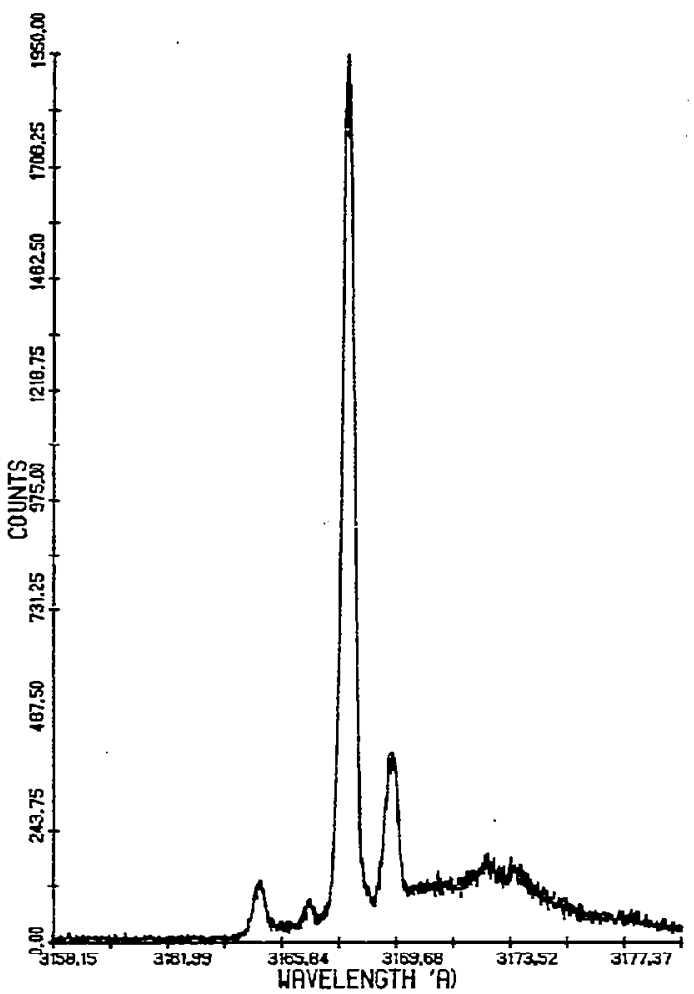

Fig. 1. Fluorescence spectrum of $2-D_{1} / D_{8}$. Fluorescence spectrum of the $9.2 \times 10^{-5} 2-D_{1} / D_{8}$ system for the " $0-0$ " emission. The main features are identified as follows: The $3168 \AA\left(31556 \mathrm{~cm}^{-1}\right)$ is the transition of the $2-D_{1}$ species to the ground state. The peak at $3169.5 \mathrm{~A}\left(31541.3 \mathrm{~cm}^{-1}\right)$ is attributed to $\mathrm{C}_{10} \mathrm{H}_{8}$ impurity present, while the two peaks at $3166.8 \AA\left(31568.8 \mathrm{~cm}^{-1}\right)$ and $3165.1 \mathrm{~A}\left(31585.4 \mathrm{~cm}^{-1}\right)$ are attributed to $D_{4}$ and $D_{5}$ impurities present. Notice that (within out experimental setup) no emission can be seen from the $C_{10} D_{8}$ species (expected at $3164.5 \AA$ or 31591.4 $\mathrm{cm}^{-1}$ ), which means that the ratio of host/suest emission should be very close to zero. This in turn produces a $P \rightarrow 1$, and a large $n(t)$ value. We did not use this particular spectrum for quantitative purposes but it nevertheless illustrates that these systems actually possess relatively large $n(t)$ values. The temperature of this spectrum is $1.8 \mathrm{~K}$.

The concentration (mole fraction) of guest determines the probability that excitation is being trapped by the guest, assuming that the trapping efficiency is equal to unity (we treat the trapping efficiency problem elsewhere [20]). It is also assumed that the excitation that is already trapped by the guest cannot

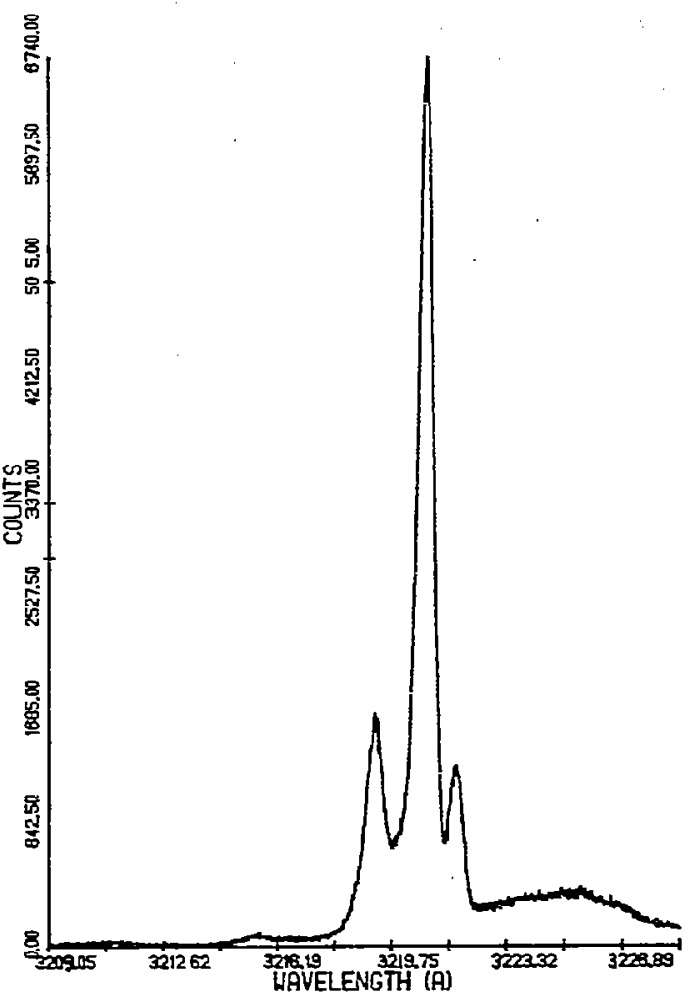

Fig. 2. Fluorescence spectrum of $1-D_{1} / D_{8}$. Fluorescence spectrum of the $8.8 \times 10^{-4} 1-D_{1} / D_{8}$ system in the " $0-512$ " $\mathrm{cm}^{-1}$ vibronic Iegion. The main features are identified as follows: The $3220.8 \mathrm{~A}\left(31038.8 \mathrm{~cm}^{-1}\right)$ is the transition to the guest ground vibronic state, while the $3219.3 \mathrm{~A}$ ( 31054.2 $\left.\mathrm{cm}^{-1}\right)$ is to the host $\left(C_{10} D_{8}\right)$ vibration at $496 \mathrm{~cm}^{-1}$. The peak at $3221.8 \mathrm{~A}\left(31030 \mathrm{~cm}^{-1}\right)$ is attributed to $\mathrm{C}_{10} \mathrm{H}_{3} \mathrm{im}$ purity present in the crystal, while the $3215.7 \AA$ peak ( $31088.5 \mathrm{~cm}^{-1}$ ) is the $C_{10} D_{8}$ emission. The guest/host ratio is calculated using the $C_{10} D_{8}$ emission for host, and the sum of all the others for the guest, since they all serve the purpose of an impurity. These measured ratios are given in table 2 . The temperature of this spectrum is $1.8 \mathrm{~K}$.

climb up the energy ladder back to the host. Because of the large energy separation $\left(5620 \mathrm{~cm}^{-1}\right.$ for the naphthalene-anthracene system) the probability amplitude for detrapping is very small. Assuming equal absorption cross sections, the probability for initial excitation by the host is given by:

$P_{\mathrm{h}}=C_{\mathrm{h}}=1-C_{\mathrm{g}}$. 
This also assumes that the excitation is being limited to one molecular site. By letting it hop to adjacent sites there is a certain number of lattices sites, $n(t)$, that are visited at least once during the lifetime of the generated excited state, and, accordingly, for $n(t)$ such sites visited, the probability $P_{h}$ for staying on the host is:

$P_{\mathrm{h}}=\left(1-C_{\mathrm{g}}\right)^{n}$.

$P_{\mathrm{g}}$, the probability for trapping by the guest, is then given by:

$P_{\mathrm{g}}=1-\left(1-C_{\mathrm{g}}\right)^{n}$,

since we require that $P_{\mathrm{h}}+P_{\mathrm{g}}=1.00$. Formula (4) is a simple minded approach used by us $[7,21]$ for such types of problems. Rearranging (4) and solving for $n(t)$ we receive:

$n(t)=\operatorname{In}\left(1-P_{\mathrm{g}}\right) / \ln \left(1-C_{\mathrm{g}}\right)$.

Because $1 \gg C_{\mathrm{g}}$ eq. (5) becomes:

$n(t)=-\ln \left(1-P_{\mathrm{g}}\right) / C_{\mathrm{g}}$.

It is seen that $n(t)$ depends only on $P_{\mathrm{g}}$ and $C_{\mathrm{g}} \cdot C_{\mathrm{g}}$ is a constant for a particular systen, and only $P_{\mathrm{g}}$ needs to be established. We do this by assuming that the probability that an excitation is trapped by impurities is measured by the fraction of the fluorescence carried by the impurity (see appendix). In fig. 3 we plot $-\ln \left(1-P_{\mathrm{g}}\right)$ versus $C_{\mathrm{g}}$ for the anthracene in naphthalene system $\doteqdot$. The slope is $n(t)=3 \times 10^{4}$, or it takes 30000 sites to be visited at least once during the lifetime for this sytem. Here, we assume that anthracene has an effective cross section of the order of unity. If we allow for trapping efficiencies $(\gamma)$ different from 1 , then eq. (6) becomes:

$n(t)=-\ln \left(1-P_{\mathrm{g}}\right) / C_{\mathrm{g}} \gamma$,

and if $\gamma>$ I then $n(t)$ would be lower. From eq. (7) we see that uncertainties in $C_{\mathrm{g}}$ affect $n(t)$ the same way as $\gamma$ does.

The temperature of the above experiments was

$\mp$ The relationship is not linear throughout the concentration range possibly because (a) at very low impurity concentration, say about $10^{-8}$ mole fraction, there is a high uncertainty for measuring such concentrations accurately, and (b) at these very low concentrations natural (intrinsic) system impurities compete with the main impurity, while at higher guest concentrations their contribution is minimal.

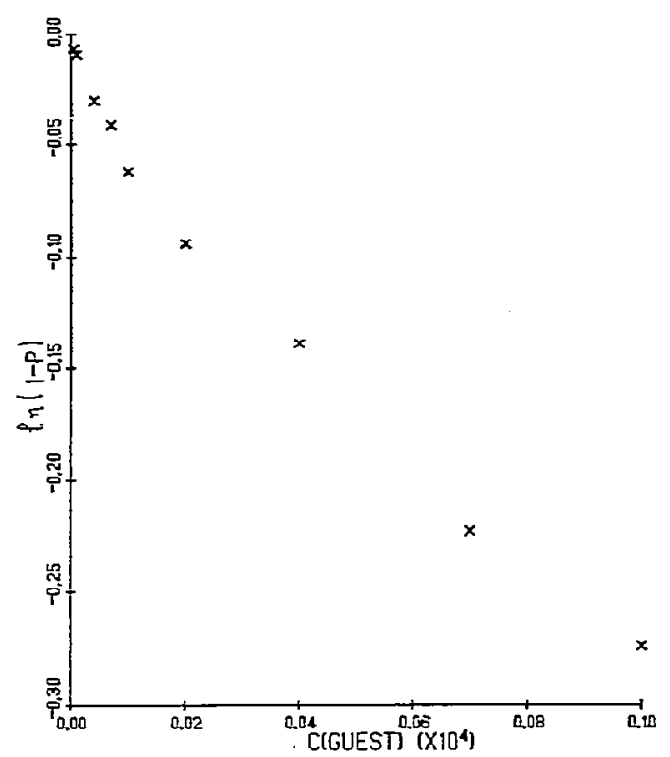

Fig. 3. Plot of $\ln \left(1-P_{\mathscr{g}}\right)$ versus $C_{\mathrm{g}}$ - The data are taken from Wolf and Port [2]. The slope in this case is used to evaluate the number of molecular sites visited in an average exciton lifetime. See text for details.

$300 \mathrm{~K}$. For lower temperatures the increase in coherence will have a serious effect on $n(t)$. We believe that this temperature effect dominates over the dependence of $\gamma$ on the temperature. This is discussed in the next section.

Similar calculations were performed for the deuterated naphthalene systems of table 2 and they yield an average $n(t)$ of about $1 \times 10^{4}$ sites. Notice, that due to the presence of $X$-traps in these systems [7], this is the absolute minimum number (lower limit) derived from the experiment, while the real number may be several times this minimum. If any of the host emission is due to $\mathrm{X}$-traps (this is probable because of the slight variation in the observed host frequency [7] of about $6-7 \mathrm{~cm}^{-1}$ ), then the actual $P$ would be higher, which would in turn produce a higher $n(t)$ value, from eq. (6). We thus get good agreement between data obtained from chemically and isotopically different guest systems, based on experiments performed in different laboratories (and as a matter of fact for different purposes!). 


\section{Correlated random walk calculations and results}

The question one would like to answer next is: How many steps does it take for an excited state to visit 30000 distinct sites in a specific period of time? In a way this is the inverse problem to the question of the number of sites visited at least once after a specific number of steps, a question that we dealt with recently [8]. Briefly, there is no unique answer to this problem since it depends on parameters such as the dimensionality of the lattice and the range and magnitude of the interactions between adjacent sites. The available exact formalism [22] deals with this problem for pure lattices, and only for jumps that exhibit total loss of memory. Also, for two-dimensional cases the analytical formalism is valid only in the limit of a very large number of steps, larger than the number of steps involved in the present work [8, $9,22]$.

We have thus developed a computer model that can accomplish these calculations. The details are described elsewhere $[8,9]$. The essential features are that we first simulate a random lattice at any desired binary concentration, including, of course, a pure lattice. We can then calculate the trapping probability by relating it to the extent of transport which is given by the number of distinct sites visited at least once during a random transfer process. Here we assume simple and irreversible trapping (for $\gamma=1$ ) when the exciton is found on a trap site, regardless of its mode of propagation and its memory, or in other words, trapping is likely at any instance when a trap site is encountered during a step of any given size. However, there is no trapping when the excitation is one or more lattice constants away from a trap site, regardless of the mode of propagation and memory. Therefore, cffectively, our trapping depends on the details of the exciton motion (not in the same fashion as suggested by Hemenger et al. [23], but somewhat equivalently). In our model, trapping probabilities are isotropic (equal in all directions) for the completely incoherent $(l=1)$ exciton motion, but not in the $l>1$ cases, where being located along the line of motion makes a site more likely to be visited than being on its side.

In table 4 we give simple random walk results for three lattices: one-dimensional linear, two-dimensional square, and three-dimensional simple cubic.
Table 4

Relation between number of steps and number of sites visited for simple random walk

\begin{tabular}{rlll}
\hline $\begin{array}{l}\text { Number } \\
\text { of steps }\end{array}$ & \multicolumn{2}{l}{ Number of sites visited } \\
\cline { 2 - 4 } & 1-dim & $\begin{array}{l}\text { 2-dim } \\
\text { square Lattice }\end{array}$ & $\begin{array}{l}\text { 3-dim } \\
\text { simple cubic }\end{array}$ \\
\hline 50000 & 357 & 12300 & 31500 \\
100000 & 505 & 23600 & 62900 \\
150000 & 618 & 34500 & 94400 \\
200000 & 713 & 44900 & 125800 \\
250000 & 798 & 58800 & 157300 \\
300000 & 874 & 70900 & 188700 \\
\hline
\end{tabular}

For the one- and three-dimensional lattices we used the exact formalism [22] and derived the number of sites visited in a random walk as a function of the number of steps. For the two-dimensional case the analytical formalism is not applicable, as discussed above. For this case we used the results from a Monte Carlo computer simulation, but the details are reported elsewhere $[8,9]$. For room temperature experiments we assume that we have a total loss of directional memory. There is good evidence [24] that for naphthalene the out-of-plane interactions are considerably lower than the in-plane ones. We thus concentrate on the 2-dimensional case. It takes about 150000 steps to visit 30000 sites; on the average, for this simple case of nearest-neighbor-only, uncorrelated random walks on a square lattice (table 4). Previously we have also performed simulations [9] on quasi-two-dimensional lattices, where the out-of-plane transfer probability was small, compared to the inplane one, and these resulted in visitation efficiencies comparable to those of the strictly two-dimensional transfer. This is different from the situation discussed by Wieting et al. [14], where a small probability of out-of-line transfer plays an important role for quasione-dimensional systems.

We row apply the above to the $300 \mathrm{~K}$ data, where we had $n(t)=3 \times 10^{4}$ sites visited, resulting in $1.5 \times$ $10^{5}$ steps. The lifetime of naphthalene is well known to be about $128 \mathrm{~ns}[16,25-27]$. This results in a mean 
jump time of about $t_{\mathrm{j}}=128 \mathrm{~ns} / 1.5 \times 10^{5}=0.9 \mathrm{ps} \div$. Wolf and Port [2] contend that $t_{j}=2$ ps at $300 \mathrm{~K}$. Thus our model is consistent, at least within an order of magnitude, with previous methods.

The effect of temperature is also of interest in this study. In table 3 we listed experimental quantum ratios as a function of temperature, from 2 to $300 \mathrm{~K}$, and we observe now that as the temperature is decreased the probability $P$ increases. We explain this trend in terms of hopping correlation or exciton quasicoherence, a type of motion which has been proposed often before [6-9]. Table 5 contains results from an earlier report [9] with calculations that include retention of the directional memory over a length of $l$ lattice units. However, the formalism [eq. (3)-(7)] remains the same as before. Theoretically, the quantum mechanical exciton-phonon (and exciton-exciton) scattering process (and its $k$-dependence) are far from fully understood [29]. It is thus not clear to us a priori whether a gaussian, lorentzian, Poisson, or other distribution of step-lengths is most appropriate. Having at our disposal extensive simulations involving gaussian distributions $[8,9]$ we used these existing simulations for binary lattices. In table 5 we show results of computer simulations with the coherence length $l$ as parameter, with a gaussian distribution of the correlation length around $l=1,10$, and 100 , with standard deviation (sd) $0,3.0$ and 30.0 , respectively. We believe that qualitatively the dependence on $l$ ("coherence length") should be similar, irrespective of the specific distribution.

Hammer's data (table 3) give $P=0.79$ at $2 \mathrm{~K}$. Thus eq. (7) gives $n(t)=44600$. At $300 \mathrm{~K}$ we have $P=0.44$ giving $n(t)=16600$ distinct sites visited. We thus have a ratio of $n(2 \mathrm{~K}) / n(300 \mathrm{~K})=2.7$. This effect can be explained qualitatively by assuming $l>1$, and probably $l=10-100$, using data of table 5 . Also, from Auweter's data at $2 \mathrm{~K}, P=0.86$, and at $300 \mathrm{~K}, P=$ 0.31 (see table 3 and appendix). These $P$ values give

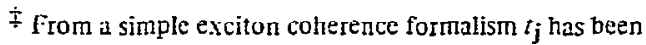
associated with the interaction between adjacent molecular sites, as $t_{\mathrm{j}}=(4.1)_{5}^{-1}$, where $M$ is this interaction energy expressed in $1 \mathrm{~Hz}$ [5]. Using [24] $M=18 \mathrm{~cm}^{-1}$ we receive $t_{\mathrm{j}}=$ 0.5 ps, again with some uncertainty because the transformation from $M$ to $t_{j}$ is not well established yet [28]. We notice that the homogencous linewidth $\lambda$ is within one order of magnitude equal to $M$ (over the entire temperature range), and that for $\Lambda \approx W$ the simple incoherent excitor formalism $t_{\mathrm{j}} \approx\left(4 M T^{2} / \Lambda\right)^{-1}$ gives again $t_{\mathrm{j}} \approx(4 M)^{-1}$.
Table 5

Effect of correlation on the number of sites visited. Square lattice simulations (sd is standard deviation in $l$ )

\begin{tabular}{llrl}
\hline $\begin{array}{l}\text { Number } \\
\text { of steps }\end{array}$ & \multicolumn{2}{l}{ Number of sites visited } \\
\cline { 2 - 4 } & $\begin{array}{l}l=1 \\
\text { sd }=0\end{array}$ & $\begin{array}{l}l=10 \\
\text { sd }=3\end{array}$ & $\begin{array}{r}l=100 \\
\text { sd }=30\end{array}$ \\
\hline 50000 & 12300 & 33300 & 36900 \\
100000 & 23600 & 66200 & 72400 \\
150000 & 34500 & 96600 & 107000 \\
200000 & 44900 & 125400 & 140400 \\
\hline
\end{tabular}

a ratio of $n(2 \mathrm{~K}) / n(300 \mathrm{~K})=5.3$. Both sets of data are consistent with greater transport efficiency (partial coherence) in the lower temperature crystals, especially the second set which is more recent (and thus presumably suffers less from the effect of quenching by shallow X-traps, due to impurities).

A second approach is to make use of the time evolution data directly. Auweter has reported [19] considerably different time curves for $7 \mathrm{~K}$ than for $300 \mathrm{~K}$. Direct integration of these curves yields a ratio of areas: $A(300 \mathrm{~K}) / A(7 \mathrm{~K})=4.0$. We assume that the natural lifetime of pure naphthalene is given by an exponential with a lifetime of $128 \mathrm{~ns}$. The actual signal we observe for the doped crystal has, of course, a shortened lifetime due to trapping. Therefore, the difference between the integrated areas of the exponential and actual naphthalene signals is the contribution of the trap signal. We measured these ratios and found them to be:

$$
\begin{aligned}
& \text { At } 7 \mathrm{~K}, A \text { (“exponential") } / A(\text { actual })=6.12, \\
& \text { at } 300 \mathrm{~K}, . \quad A(\text { ("exponential") } / A(\text { actual })=1.53 \text {. }
\end{aligned}
$$

From these ratios we can get $P$ values (see appendix) using: $P=A$ ("exponential" - "actual")/A("exponentia!"). We get: $P=0.84\left(\right.$ at $7 \mathrm{~K}$ ) and $n(t)=3.5 \times 10^{5}$ sites, and $P=0.35$ (at $300 \mathrm{~K}$ ) with $n(t)=8.1 \times 10^{4}$ sites, or a ratio $n(7 \mathrm{~K}) / n(300 \mathrm{~K})=4.3$, again pointing out the necessity of coherence to explain the changes in this ratio with temperature. We note that while the absolute values of $n(t)$ may only be order-of-magnitude indications, the relative data appear to be more reliable. We also note the good agreement between the two methods of obtaining $P, n(t)$, and the temperature effect. 


\section{Discussion and conclusions}

It has been pointed out by Auweter that the above temperature dependence of the energy transport can be explained in terms of the trapping cross section of anthracene [19]. At low temperature anthracene has an effective cross section greater than unity because of the "funnelling-in" of excitons from nearby adjacent sites, while at higher temperatures the effect of this funnel is reduced due to detrapping. However, we do not expect energy "funnels" for isotopically substituted naphthalenes acting as traps (as we discussed earlier). We believe that the actual observed temperature effect may be in part due to some effective efficiency greater than unity at low temperatures which decreases towards room temperature, but we suggest that the coherence of exciton motion plays an important, and probably dominant role. At least qualitatively the trends exhibited by the data referred to here agree with the model (loss of coherence) we presented earlier. We thus basically adopt the earlier interpretation of the Stuttgart group $[2,18]$ without though relying on a specific model of exciton coherence [30], and without changing the mean hopping time with temperature.

We described an energy transfer hopping model that has made it possible to arrive at order-of-magnitude estimates for the number of jumps and applied it to the first singlet excited state of naphthalene crystals. We made use of doped crystal systems and developed a combination of experimental optical emission intensities and statistical simulations on random lattices to examine the various ways of energy propagation. We considered both the sinple random walk case and a coherent (correlated) mode of transfer. A simple expression was obtained that gives the number of hops in terns of experimental observables, and it was applied to the various systems discussed earlier. Our results show that within one order of magnitude (the uncertainty being attributed to the differences in trapping efficiencies) the same characteristics pertain to different host and guest species both at low ( $2 \mathrm{~K}$ ) and high (room) temperatures. The effect of the exciton-phonon coupling, which increases with temperature, is to shorten the coherence lengths. This alters the results of the model by only one order of magnitude in $n(t)$. Therefore, it is not intended to use this approach for the derivation of coherence lengths but rather to derive estimates for the number of sites visited during the lifetime of the excitation. The agreement between the different systems is rather satisfactory, considering the various factors discussed earlier. We arrive at an energy transfer time between two adjacent molecules of the order of 1 ps. Wolf and Port [2] gave an educated guess for the same quantities we calculated, without making full use of random walk simulations. These are in agreement with ours. Powell and Soos [1] used a different technique, but they derived results differing by two or more orders of magnitude from ours. In particular they report a hopping time of $t_{j}=200 \mathrm{ps}$ for naphthalene (and $t_{\mathrm{j}}=30 \mathrm{ps}$ for anthracene), which corresponds to a total number of only 100 to 1000 steps, compared to our $10^{5}$. They derive these results from time resolved spectra by plotting the behavior of versus time $k(t)$, the time dependent rate constant. Then, they try to fit this $k(t)$ to an empirical relation of the type:

$k(t)=N_{\mathrm{A}}\left(a+b t^{1 / 2}\right)$.

where $N_{\mathrm{A}}$ is the concentration of the guest species and $a$ and $b$ are constants. They get a good fit for "unreasonably large values of $R_{0}$, on the order of $30-$ $100 \AA$ ", where $R_{0}$ is a radius of a sphere of an impurity center. They are thus forced to assume the existence of a funnel-type region, even at room temperatures. Auweter's suggestion is that only at low temperatures is such a funnel of importance. As it can be seen from eq. (7) any $\gamma>1$ will reduce $n(t)$ by the same factor $(\gamma)$, but a difference of 2 or 3 orders of magnitude can hardly be explained by differences in trapping efficiencies only. Even for the uncorrelated random walk case there are serious questions pertaining to the Powel and Soos model [31]. In addition we believe that one has to consider the coherence of motion as well.

\section{Note added in proof}

Both experiments (naphthalene X-traps absorption spectra) and theoretical considerations (local perturbation versus exciton bandwidth) seem to be inconsistent with a large-radius "exciton funnel" surrounding the anthracene guest at low temperature (S.T. Gentry, D.W. Hooper and R. Kopelman, unpublished). 


\section{Appendix}

The impurity trapping probability $P$ can be related to the energy trapping rate constant as follows: The radiative quantum ratio of anthracene to naphthalene $Q_{\mathrm{A}} / Q_{\mathrm{N}}$ is given by:

$Q_{\mathrm{A}} / Q_{\mathrm{N}}=k_{\mathrm{A}} N_{\mathrm{A}} / k_{\mathrm{N}} N_{\mathrm{N}}$,

where $k_{\mathrm{A}}$ and $k_{\mathrm{N}}$ are the radiative decay rates of anthracene and naphthalene, respectively. Manipulation of this equation yields $(q \equiv Q / \phi)$.

$$
\begin{aligned}
P & =q_{\mathrm{A}} /\left(q_{\mathrm{A}}+q_{\mathrm{N}}\right) \\
& =\phi_{\mathrm{N}} k_{\mathrm{A}} N_{\mathrm{A}} /\left(\phi_{\mathrm{A}} k_{\mathrm{N}} N_{\mathrm{N}}+\phi_{\mathrm{N}} k_{\mathrm{A}} N_{\mathrm{A}}\right) .
\end{aligned}
$$

The rate equation for the anthracene exciton population [32] is:

$\mathrm{d} N_{\mathrm{A}} / \mathrm{d} t=-K_{\mathrm{A}} N_{\mathrm{A}}+k_{\mathrm{WG}} N_{\mathrm{N}}$.

where $k_{W G}$ is the energy transfer rate constant, and $K_{\mathrm{A}}$ is the total (radiative + nonradiative) rate constant $\left(K_{\mathrm{A}}=k_{\mathrm{A}} / \phi_{\mathrm{A}}\right)$. At steady-state conditions the left side vanishes yielding $K_{\mathrm{A}} N_{\mathrm{A}}=k_{\mathrm{WG}} N_{\mathrm{N}}$. Using now the equation for $P$ we receive:

$1 / P=1+k_{\mathrm{N}} / \dot{\phi}_{\mathrm{N}} k_{\mathrm{WG}}=1+K_{\mathrm{N}} / k_{\mathrm{WG}}$.

We note that this equation is valid also under nonsteady state conditions, provided that $k_{W G}$ is a real rate constant (time independent), i.e. in the SternVolmer limit [31].

In the general case where $k_{w G}$ is time dependent, one can still obtain $P$ from the decay curves of the host (naphthalene) via the following procedure: (1) normalization to unity of the decay rate at $t=0$ for both pure host (naphthalene) and for the doped system and then integrating the areus under the curves; (2) subtraction of the two integrated areas and division by the pure host area. The only assumptions underlying this method are: (a) There is no other decay channel (e.g. fusion, defect trapping). (b) The extrapolation to $t=0$ is justified (i.e. no drastic change in the decay rates during the first few nanoseconds). We note that instead of measuring the decay curve of a really pure and perfect crystal (naphthalene) we believe that it is sufficient to plot the (exponential) decay rate for the naphthalene excitation as derived from lifetime measurements on naphthalene (dilute) in a perdeuteronaphthalene host [16].

\section{References}

[1] R.C. Powell and Z.G. Soos, J. Luminescence 11 (1975) 1.
[2] H.C. Wolf and H. Port, J Luminescence 12/13 (1976) 33.

[3] R. Silbey, Ann. Rev. Phys. Chem. 27 (1976) 203.

[4] A.H. Francis and R. Kopelman, in: Topics in applied Physics, eds. W.M. Yen and P.M. Selzer (Springer, Berlin, 1980).

[5] R.Kopelman, in: Topics in applied physics, Vol. 15, ed. F.K. Fong (Springer, Berlin, 1976) p. 297.

[6] P. Argyrakis and R. Kopelman, J. Chem. Phys. 66 (1977) 3301.

[7] P. Argyrakis, Doctoral Dissertation, University of Michigan (1979).

[8] P. Argyrakis and R. Kopelman, Phys. Rev. B, to be published.

[9] P. Argyrakis and R. Kopelman, J. Theoret. Bio 73 (1978) 205.

[10] V.M. Kenkre and R.S. Knox, Phys. Rev. B 9 (1974) 5279; J. Luminescence 12/13 (1976) 187.

[11] M. Grover and R. Silbey, J. Chem. Phys. 54 (1971) 4843.

[12] H. Haken and R. Reineker, Z. Phys. (1972) 249, 253.

[13] H. Haken and G. Strobl, Z. Phys. (1973) 262, 135.

[14] R.D. Wieting, M.D. Fayer and D.D. Dlott, J. Chem. Phys. 69 (1978) 1996.

[15] R.M. Shelby, A.H. Zewail, and C.B. Harris, J. Chem. Phys. 64 (1976) 3192;

C.B. Harris and M.D. Fayer, Phys. Rev. B, 10 (1974) 1784;

D.M. Burland and A.H. Zewail, Advan. Chem. Phys. 40 (1979) 369.

[16] D.C. Ahigren, Doctoral Dissertation, University of Michigan (1979).

[17] A. Hammer, Doctoral Dissertation, University of Stuttgart (1968).

[18] A. Hammer and H.C. Wolf, Mol. Cryst. 4 (1968) 191.

[19] H. Auweter, Doctoral Dissertation, University of Stuttgart (1978).

[20] P. Argyrakis, D. Hooper and R. Kopelman, in preparation.

[21] J. Hosken, private communication (1975).

[22] E.W. Montroll, Proc. Symp. Appl. Math. 16 (1964) 193.

[23] R.P. Hemenger, K. Lakatos-Lindenberg and R.M. Pearlstein, J. Chem. Phys. 60 (1974) 3271; J. Math. Phys. 13 (1972) 1056 .

[24] H.-K. Hong and R. Kopalman, 3. Chem. Phys. 55 (1971) 724.

[25] T.B. El-Kareh and H.C. Wolf, Mol. Cryst. 4 (1968) 195.

[26] K. Uchida and M. Tomura, J. Phys. Soc. Japan 36 (1974) 1358.

[2]] M. Kohler, D. Schmid and H.C. Wolf, J. Luminescence 14 (1976) 41.

[28] D.M. Hanson, Preprint (1975).

[29] D.P. Craig and L.A. Dissado, Chem. Phys. 14 (1976) 89; D.P. Craig, S.H. Walmsley and L.A. Dissado, Chem. Phys. 46 (1980) 887.

[30] V.M. Agranovich and Y.V. Konobeev, Soviet Phys. Solid State 5 (1963) 999.

[31] R. Kopelman and P. Argyrakis, J. Chem. Phys. 72 (1980) 3053 .

[32] P. Argyrakis and R. Kopelman, Chem. Phys. Letters 61 (1979) 187. 